\title{
DUST IN PLANETARY NEBULAE AND IN POST-AGB OBJECTS
}

\author{
M. J. BARLOW \\ Department of Physics $\&$ Astronomy, University College London, \\ Gower Street, London WC1E 6BT, U.K.
}

\begin{abstract}
Developments in the field since the review of Roche (1989) are discussed. Section 1 describes the results from detailed models of the dust emission from planetary nebulae and summarises the derived dust-to-gas mass ratios. The properties of the remarkable gas-poor planetary nebula IRAS 18333-2357, located in the globular cluster M22, are discussed in Section 2. The use of thermal infrared imaging to map the distribution of different dust components is reviewed in Section 3, and two recently applied optical probes of nebular dust properties are described: emission line spectropolarimetry, and spectroscopy of the Extended Red Emission (ERE). The final section describes recent results from infrared spectroscopy. The spectra of carbon-rich postAGB objects have provided valuable new insights into the nature and evolution of the materials responsible for the UIR bands, while unusual oxide grains and crystalline ice emission have been detected around oxygen-rich post-AGB objects. The spectra of a small number of objects show evidence for the simultaneous presence of both oxygen-rich and carbon-rich material.
\end{abstract}

\section{Dust to Gas Mass Ratios in Planetary Nebulae}

Harrington, Monk \& Clegg (1988) modelled the dust emission from the carbonrich nebula NGC 3918 using graphite and amorphous carbon grain models with power-law size distributions of the type discussed by Mathis, Rumpl \& Nordsieck $(1977 ; \mathrm{MRN})$. Because graphite grains have a steeper infrared emissivity law and are less efficient emitters, they will be hotter than amorphous carbon grains of the same size. To fit the overall energy distribution, Harrington et al. had to have a lower limit of $0.04 \mu \mathrm{m}$ for the radius of graphite grains (compared to $0.005 \mu \mathrm{m}$ for the standard MRN insterstellar grain mixture), since grains smaller than this produced too much flux at $10 \mu \mathrm{m}$ and shorter IR wavelengths. On the other hand, their amorphous carbon model required a lower grain radius cut-off of $0.0005 \mu \mathrm{m}$ in order to produce enough $10 \mu \mathrm{m}$ flux. Both grain models had maximum grain radii of $0.25 \mu \mathrm{m}$, similar to the MRN insterstellar mixture. The graphite and amorphous carbon grain models for NGC 3918 implied overall dust-to-gas mass ratios of $8 \times 10^{-4}$ and $3 \times 10^{-4}$, respectively. Harrington et al. noted that if the observations had been modelled assuming single-temperature blackbody emission (as is sometimes found in the literature), a misleadingly high dust-to-gas mass ratio of $2 \times 10^{-3}$ would have been derived.

Hoare, Roche \& Clegg (1992) have modelled the overall dust emission from the C-rich PN NGC 7027 and BD $+30^{\circ} 3639$, again using both graphite and amorphous carbon models but this time also utilizing their own JCMT sub-mm and mmwavelength photometry as well as the IRAS and shorter wavelength infrared data. For both PN they found that their graphite grain models were unable to produce enough sub-mm flux but that an acceptable fit could be obtained with amorphous carbon models. Figure 1 shows their fit to the IR and sub-mm energy distribution of NGC 7027. The dotted line shows the predicted emission from dust in the ionized zone - the remaining emission comes from dust in the surrounding neutral zone. 


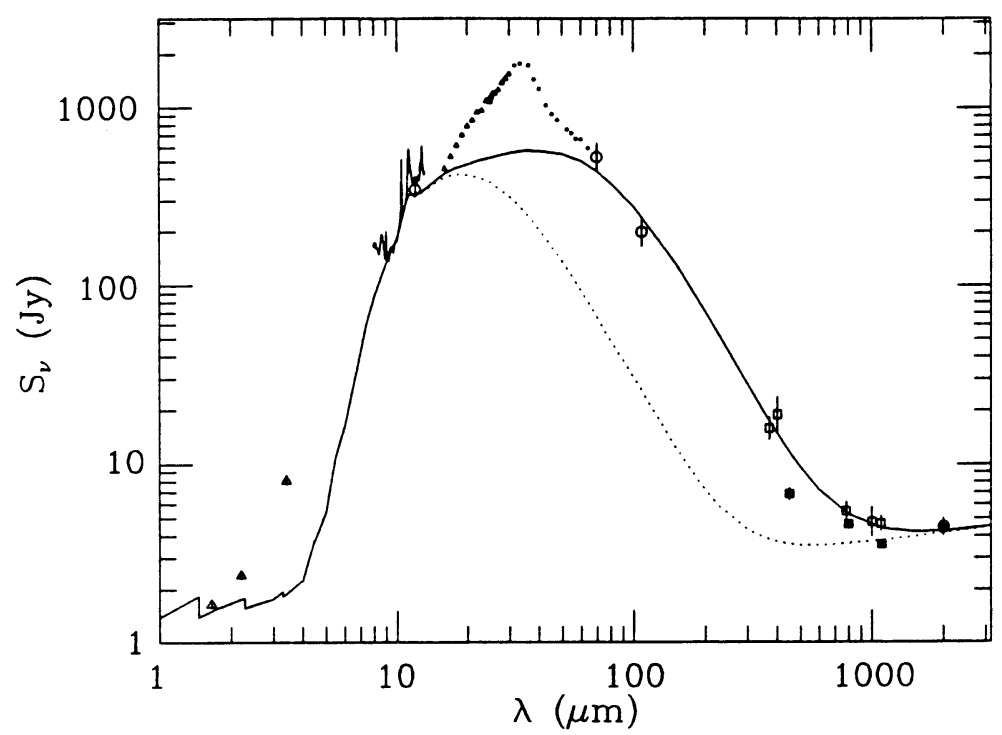

Fig. 1. The observed infrared energy distribution of NGC 7027 is shown, along with an amorphous carbon grain model fit (solid line). The dotted line shows the predicted emission from the dust in the ionized zone alone. From Hoare, Roche \& Clegg (1992).

They estimated a dust-to-gas mass ratio of $7 \times 10^{-4}$ in the ionized zone, based on the observed attenuation of the C IV $1550 \AA$ resonantly scattered line (use was made of the Middlemass (1990) photoionization model of NGC 7027, which predicted the flux emitted in this line before dust attenuation). For the neutral zone around NGC 7027 , Hoare et al. estimated a dust-to-gas mass ratio of $1.5 \times 10^{-3}$, not significantly different from the ratio derived for the ionized zone in view of the uncertainties associated with the CO-based neutral mass estimate. An amorphous carbon dust visual optical depth of 0.4 was estimated for the neutral zone.

Inspection of Figure 1 shows that there is a very prominent feature peaking at $30-35 \mu \mathrm{m}$ which is not fitted by the model (Hoare et al. used unpublished $K A O$ spectrophotometry by Moseley, Glaccum \& Silverberg). This $30 \mu \mathrm{m}$ feature had previously been observed in the spectra of carbon stars and IC 418 (Forrest et al. 1981; Moseley \& Silverberg 1986) and an identification with solid MgS has been discussed by Goebel \& Moseley (1985). However, in the case of IC 418 about $25 \%$ of the total IR luminosity is emitted in the $30 \mu \mathrm{m}$ band (the feature is similarly prominent in NGC 7027; Fig. 1). It would seem surprising if material composed of relatively low abundance elements such as $\mathrm{Mg}$ and $\mathrm{S}$ could emit such a large fraction of the total flux. Further, Middlemass (1988) found magnesium to be undepleted in the nebular gas phase in IC 418. Given the high abundance of carbon in all the objects so far found to emit the $30 \mu \mathrm{m}$ feature, a carbonaceous origin for the feature would seem more likely. There is also an excess over the model predictions in the $2-5 \mu \mathrm{m}$ region of the spectrum of NGC 7027 (Figure 1). This phenomenon has been 
noted in other PN too and temperature spiking in small grains has been suggested as the origin for this short-wavelength excess.

\section{TABLE I}

Dust-to-Gas Mass Ratios in the ionized zones of C-rich PN

$\begin{array}{lcccl}\text { Name } & \mathrm{C} / \mathrm{O} & \mathrm{n}_{e}\left(\mathrm{~cm}^{-3}\right) & \mathrm{M}_{\text {dust }} / \mathrm{M}_{\text {gas }} & \text { Reference } \\ \text { NGC 7027 } & 2.3 & 50000 & 7 \times 10^{-4} & \text { Hoare et al. (1992) } \\ \text { BD+30 } 3639 & 2.0 & 15600 & 7 \times 10^{-4} & \text { Hoare et al. (1992) } \\ \text { IC 418 } & 1.3 & 9000 & 6 \times 10^{-4} & \text { Hoare (1990) } \\ \text { NGC 3918 } & 1.6 & 6000 & 3 \times 10^{-4} & \text { Harrington et al. (1990) } \\ \text { NGC 7662 } & 1.7 & 2500 & 3 \times 10^{-4} & \text { Hoare (1990) }\end{array}$

Table I collects together all the dust-to-gas mass ratios that have been derived to date for the ionized zones of carbon-rich PN. They were all derived from detailed photoionization plus dust modelling, using amorphous carbon grains with MRN size distributions. The mean nebular electron densities for the five PN span a range of a factor of twenty $\left(50000 \mathrm{~cm}^{-3}\right.$ down to $2500 \mathrm{~cm}^{-3}$, yet the derived dust-to-gas mass ratios decrease by no more than a factor of two over the same range. There does not therefore seem to be evidence for significant destruction of dust after incorporation into the ionized region of PN. The highest PN dust-to-gas mass ratios in Table I are about a factor of ten lower than the ratio of $7 \times 10-3$ that is believed to prevail in the general interstellar medium (MRN).

\section{IRAS 18333-2357, a Remarkable Planetary Nebula in M22}

The infrared source IRAS 18333-2357 near the centre of the globular cluster M22 $(\mathrm{D}=3 \mathrm{kpc}$ ) was identified as a planetary nebula by Gillett et al. (1989). The nebula is remarkable in (a) being bow-shaped, with an off-centre exciting star $(\mathrm{V}=14.3)$, apparently as a result of the cluster's motion through the interstellar medium; and (b) in showing no detectable hydrogen or helium emission lines, its spectrum being dominated by [O III] and [Ne III] lines. In this latter respect it shows similarities to the inner nebulosities of A30 and A78. From ionized gas mass estimates based on the forbidden lines, and from dust mass estimates based on silicate or amorphous carbon optical constants, Gillett et al. derived a dust-to-gas mass ratio of approximately unity for the nebula, implying equal quantities of heavy elements in the gas and in the grains.

Borkowski \& Harrington (1991) have carried out detailed modelling of the gas and dust emission from IRAS $18333-2357$. The $50000 \mathrm{~K}$ central star was found to have a luminosity of $3680 \mathrm{~L}_{\odot}$, with $600 \mathrm{~L}_{\odot}$ being absorbed and re-emitted in the infrared by the dust. Because of the severe deficiency of hydrogen and helium in the nebula, they found that they were unable to account for the observed optical forbidden line strengths based on the normal nebular heating mechanism of pho- 
toelectron ejection from $\mathrm{H}$ and He during photoionization. Instead they were able to show that photoelectron ejection from the high relative abundance dust grains can heat the nebular gas to temperatures of $\sim 10^{4} \mathrm{~K}$ and account for the observed optical forbidden lines. The $\mathrm{C} / \mathrm{O}$ ratio in the nebula is not well-constrained and Borkowski \& Harrington opted for amorphous carbon grains on the grounds that a low metallicity giant star could not produce a high abundance of silicate grains, whereas a high mass in carbon grains would be feasible following helium-burning and the 3rd dredge-up. Graphite and vitreous carbon have well-characterized photoelectron yield curves and Borkowski and Harrington obtained a good fit to the nebular observations using these yields. This is of significance for interstellar medium studies as a whole because photoelectron ejection from grains by UV photons (first proposed by Watson 1972) is believed to be the dominant heating mechanism for diffuse interstellar clouds - IRAS $18333-2357$ has provided direct proof that this heating mechanism operates as hypothesized.

\section{The Spatial Distribution of Dust}

As reviewed by Roche (1989), near-IR array observations have for some time made it possible to image the distribution of emission in the $3.3 \mu \mathrm{m}$ UIR ('unidentified infrared') band in PN and other objects. The advent of mid-infrared (10 and $20 \mu \mathrm{m})$ arrays has made it possible to directly probe the thermal emission from the main grain components, by imaging in bandpasses centred on the continuum and on the main dust features (e.g. the silicate, silicon carbide and UIR bands). These maps can be compared with the distribution of ionized gas mapped using mid-IR lines such as [Ne II] or [S IV], or with radio maps of the nebular free-free emission. The mid-infrared continuum emission originates from the warmer dust immersed in the ionized regions - imaging at far-IR wavelengths would be necessary to reveal the cooler dust continuum associated with neutral material surrounding a PN. In the case of planetary nebula precursors (post-AGB objects) which are not yet ionized, infrared imaging can provide the sole means of determining the size and morphology of the extended material.

Hora et al. (1990) have presented high resolution $\left(0.78 \operatorname{arcsec}\right.$ pixel $^{-1} 16 \times 16$ pixel images of NGC 6572 and $\mathrm{BD}+30^{\circ} 3639$ in five mid-IR passbands. NGC 6572 is known to emit a strong $10.5-12.5 \mu \mathrm{m} \mathrm{SiC} \mathrm{emission} \mathrm{feature.} \mathrm{Hora} \mathrm{et} \mathrm{al.} \mathrm{found} \mathrm{that}$ their $11.2 \mu \mathrm{m}$ image (which would include $11.25 \mu \mathrm{m}$ UIR band emission, if present) showed a stronger and more extended eastern lobe than did their $12.36 \mu \mathrm{m}$ image (which should probe just the SiC emission). They concluded that $11.25 \mu \mathrm{m}$ UIR band emission was responsible for the difference between the two images. Mid-infrared imaging capabilities are developing very rapidly, as evidenced by the number of posters presenting new results at this meeting. Mid-IR images of planetary nebulae are presented by Hora et al. (Poster III-75), Meixner et al. (Poster III-76) and Deutsch et al. (Poster III-125), while mid-IR imaging data on post-AGB objects and on AGB stars are presented by Meixner et al. (Poster III-124) and by Skinner et al. (Poster III-102), respectively. 
It is also possible to map dust spatial distributions at optical wavelengths. Only a very few PN have a high enough circumnebular dust optical depth for reflection nebulae to be detected or extinction variations to be mapped (e.g. NGC 7027: Middlemass, Clegg \& Walsh 1989; NGC 6302: Ashley 1990) but a number of other optical methods are available for mapping dust distributions in PN. Walsh \& Clegg (Poster III-98) have measured polarized [O III] and $\mathrm{H} \alpha$ line profiles from six PN at high spectral resolution. At the nebular centres they found the polarization to be very low or undetectable at the line peaks but to increase to $\sim 5 \%$ in the line wings, with stronger polarization in the red wing. The light scattered from the neutral halo around NGC 7027 was found to be polarized by up to $35 \%$. Walsh and Clegg interpreted their results in terms of single-scattering 'moving mirrors' dust models. The emission seen at line centre is predominantly unscattered and so the polarization is low. Since the atoms and dust grains in an expanding nebula are red-shifted with respect to each other, photons will be predominantly scattered in the red wings of lines, where the intrinsic emission is lower, so the degree of polarization will be higher.

Furton \& Witt (1990) have detected and mapped the spatial distribution of extended red emission (ERE) around NGC 7027, using long-slit spectrophotometry. This emission, which was first discovered around the C-rich post-AGB object HD 44179 (the Red Rectangle), manifests itself as a very broad luminescence band extending from $6000 \AA$ to longer than $8000 \AA$. The ERE from the Red Rectangle shows a highly structured spectrum (Schmidt, Cohen \& Margon 1980) and the objects in whose spectrum it has been detected all exhibit the $3.3 \mu \mathrm{m}$ and longer wavelength UIR bands. The ERE is therefore believed to be arise from hydrocarbons, with hydrogenated amorphous carbon (HAC) being one candidate (Duley 1985). Furton \& Witt (1990) carefully allowed for nebular continuum emission processes in deriving the spectral shape and strength of the ERE in NGC 7027. They found that the strength of the ERE peaked just beyond the ionized boundary of NGC 7027 and was perhaps more extended than the $3.3 \mu \mathrm{m}$ UIR band emission. Furton \& Witt (1992) have observed twenty more PN in the same manner, positively identifying ERE in the spectra of seven of them, all of which had $\mathrm{C} / \mathrm{O}$ ratios in excess of unity. However, they noted several PN in whose spectra UIR band emission had been detected but for which they only obtained ERE upper limits (J900, NGC 3242 and NGC 6543).

\section{Infrared Spectroscopy of Planetary Nebulae and Post-AGB Objects}

One of the most important developments since the last meeting has been the identification of a significant number of sources from the IRAS database with post-AGB objects (e.g. Manchado et al. 1989, van der Veen, Habing \& Geballe 1989, Hrivnak, Kwok \& Volk 1989). These are objects whose optical counterparts typically have $\mathrm{F}$ or G-type supergiant spectra and are believed to be transiting from the cool AGB phase to the hot planetary nebula phase, the timescale for which should be at most a few thousand years. Since they are not yet hot enough to ionize the 
material around them, they were only recognized as potential post-AGB objects by virtue of the large infrared dust excesses detected by the IRAS survey. These objects are of great interest since the dust around them is situated in a completely different physical environment from that encountered in either the AGB or PN phases and so spectroscopy can potentially reveal new aspects of the dust properties. Unlike AGB stars, which have a fairly continuous energy distribution resulting from the attenuation of their photospheric distributions by hot dust which re-emits in the near-IR and longer wavelengths, these post-AGB objects are characterized by 'double-peaked' energy distributions (Hrivnak et al. 1989). This is because the dust created during the high mass loss rate AGB phase has moved out and cooled to fairly low temperatures $(100-200 \mathrm{~K})$ whose emission peaks at $10-20 \mu \mathrm{m}$, leaving a dip at near-IR wavelengths because of the relative lack of hotter dust particles. It is convenient to discuss separately the carbon-rich $(\mathrm{C} / \mathrm{O}>1)$ and oxygen-rich $(\mathrm{C} / \mathrm{O}<1)$ objects because of the completely different infrared spectra that they present.

\subsection{Carbon-Rich Objects}

Roche (1989) has presented the statistics for PN of the incidence of the various midIR dust features (silicates, silicon carbide, UIR bands) versus the nebular $\mathrm{C} / \mathrm{O}$ ratio, which showed that silicates are associated with nebulae having $\mathrm{C} / \mathrm{O}<1$, whereas $\mathrm{SiC}$ emission occurs in nebulae with $\mathrm{C} / \mathrm{O} \geq 1$. Strong UIR band emission is in general associated with $\mathrm{PN}$ having $\mathrm{C} / \mathrm{O}>2$ (Barlow 1983). Cohen et al. (1989) have shown that there is a very good correlation between the nebular $\mathrm{C} / \mathrm{O}$ ratio and the fraction of the total infrared flux appearing in the $7.7 \mu \mathrm{m}$ band (a feature attributed to C-C stretches in aromatic hydrocarbons; Allamandola et al. 1989).

Kwok, Volk \& Hrivnak (1989) discovered a strong broad $21 \mu \mathrm{m}$ emission band in the IRAS LRS spectra of four carbon-rich post-AGB stars, the first features to be found in the $20 \mu \mathrm{m}$ spectral region of objects exhibiting the mid-infrared UIR bands, and emitted by the coolest objects yet found with UIR bands (spectral types F3I to G5I). Buss et al. (1990) showed that the UV photon fluxes from these F-G stars are insufficient to pump the observed infrared bands, implying that they must be excited by optical photons. Buss et al. obtained $5-13 \mu \mathrm{m}$ spectra of two of Kwok et al.'s sources and found $6.2 \mu \mathrm{m}, 6.9 \mu \mathrm{m}$ and $8.0 \mu \mathrm{m}$ emission features, but with the $6.9 \mu \mathrm{m}$ feature much stronger relative to the $6.2 \mu \mathrm{m}$ feature than in hot UV-emitting objects such as NGC 7027. In addition, the strong $7.7 \mu \mathrm{m}$ feature attributed to aromatic C-C stretching was shifted longwards to $8.0 \mu \mathrm{m}$. While both the $6.2 \mu \mathrm{m}$ and $7.7 \mu \mathrm{m}$ features are normally attributed to aromatic $\mathrm{C}-\mathrm{C}$ stretch modes, the $6.9 \mu \mathrm{m}$ feature was attributed by Buss et al. to $\mathrm{CH}$ deformation modes in $\mathrm{CH}_{2}$ and $\mathrm{CH}_{3}$ groups cross-linking aromatic domains. They also found evidence for strong underlying plateau emission in the $6-9 \mu \mathrm{m}$ and $10-13 \mu \mathrm{m}$ regions and associated these with clusters of PAH molecules containing up to 500 carbon atoms. They suggested that such clusters could more easily survive in the less harsh radiation environment around $\mathrm{F}$ and $\mathrm{G}$ type stars compared to PN. As well as the plateau emission, Buss et al. proposed that these clusters could produce the $21 \mu \mathrm{m}$ emission feature by 
means of aromatic out-of-plane ring bending modes. However, Allamandola et al. (1989) predicted such modes to be significantly weaker than the modes responsible for the shorter wavelength features, whereas the reverse appears to be the case for the $21 \mu \mathrm{m}$ band sources. Sourisseau, Coddens \& Papoular (1992) have suggested that the $21 \mu \mathrm{m}$ band is due to $\mathrm{N}-\mathrm{C}-\mathrm{N}$ and $\mathrm{O}-\mathrm{C}-\mathrm{N}$ deformation modes of amides such as urea.

Justtanont, Barlow \& Skinner (Poster IV-122) have obtained 10 and $20 \mu \mathrm{m} \mathrm{spec-}$ tra at a resolving power of $\mathrm{R}=60-80$ for three of the Kwok et al. $21 \mu \mathrm{m}$ band sources. They resolve up to six separate peaks in the $7.5-13.3 \mu \mathrm{m}$ region. The $11.2-$ $11.3 \mu \mathrm{m}$ feature normally attributed to out-of-plane $\mathrm{C}-\mathrm{H}$ bending modes is shifted to $11.55 \mu \mathrm{m}$ and the strong $12 \mu \mathrm{m}$ 'plateau' seen in the lower resolution spectrum of Buss et al. is found to contain three other peaks, at $10.7 \mu \mathrm{m}, 12.2 \mu \mathrm{m}$ and $12.7 \mu \mathrm{m}$, the former two not having been clearly observed anywhere else before. The overall $10.5-13.5 \mu \mathrm{m}$ plateau observed in UIR band sources has been attributed by Allamandola et al. to bending modes of (with increasing wavelength) nonadjacent, doubly-adjacent and triply-adjacent peripheral $\mathrm{H}$ atoms on the surfaces of polycyclic aromatic hydrocarbon (PAH) clusters. Justtanont, Barlow \& Skinner found the $21 \mu \mathrm{m}$ band in the spectra of IRAS $04296+3429$ and IRAS $22272+5435$ to be be resolved into regularly spaced peaks at $20.45 \mu \mathrm{m}, 21.05 \mu \mathrm{m}, 21.7 \mu \mathrm{m}$ and $22.4 \mu \mathrm{m}$, with an energy spacing of about $14 \mathrm{~cm}^{-1}$ between successive peaks. Omont (1993) has presented $K A O$ spectra which show that two of the $21 \mu \mathrm{m}$ band sources have even stronger $30 \mu \mathrm{m}$ emission bands.

Roche, Aitken \& Smith (1991) published $7.7-13.2 \mu \mathrm{m}$ spectra at $\mathrm{R}=110$ of a number of PN and post-AGB objects that exhibit UIR-band spectra. They found the asymmetric profile of the $11.25 \mu \mathrm{m}$ band to vary significantly from object to object, with a few objects exhibiting a subsidiary peak at $11.05 \mu \mathrm{m}$. In their $\mathrm{R}=400$ spectra of three PN and one post-AGB object, Witteborn et al. (1989) found the ' $11.3 \mu \mathrm{m}$ ' feature to peak at $11.22 \mu \mathrm{m}$. Tokunaga et al. (1988) presented $\mathrm{R}=1500$ spectra of NGC 7027 and the Red Rectangle, which showed the Red Rectangle $3.29 \mu \mathrm{m}$ band to be significantly the narrower and to peak at a slightly longer wavelength. Geballe et al. (1989) found the Red Rectangle to have a relatively weak and broad subsidiary $3.4 \mu \mathrm{m}$ feature at the stellar position but that this feature increased in prominence at $5 " \mathrm{~N}$ from the central star, where it showed a $3.4-3.6 \mu \mathrm{m}$ band structure similar to that observed in UIR band sources such as NGC 7027 (e.g. Nagata et al. 1988). The $3.29 \mu \mathrm{m}$ band is normally attributed to aromatic C-H $\mathrm{v}=1-0$ stretch vibrations. However, the subsidiary bands in the $3.4-3.6 \mu \mathrm{m}$ region have been alternatively identified with (a) $\mathrm{C}-\mathrm{H}$ vibrations of aliphatic sidegroups attached to PAH structures (de Muizon et al. 1986); or (b) to higher vibrational aromatic C-H stretch transitions (e.g. $\mathrm{v}=2-1, \mathrm{v}=3-2$ 'hot bands') which are shifted from the $\mathrm{v}=1-0$ transition by anharmonic effects (Allamandola et al. 1989). The discovery that the $3.4-3.6 \mu \mathrm{m}$ 'sidebands' are stronger than the $3.29 \mu \mathrm{m}$ band in the spectrum of the G-supergiant post-AGB source IRAS $05341+0852$ (Geballe \& van der Veen 1990), as well as in the spectra of two of the G-supergiant post-AGB objects which have the $21 \mu \mathrm{m}$ band (Geballe et al. 1992), rules out 'hot bands' as the 
origin of the side-bands in these sources. For these sources at least, interpretation (a) of the $3.4-3.6 \mu \mathrm{m}$ bands as due to aliphatic sidegroups attached to aromatic clusters appears to be the most viable hypothesis at present. This also accords with the interpretation above that the strong $6.9 \mu \mathrm{m}$ feature and the $10.5-13.5 \mu \mathrm{m}$ plateau in the spectra of the $21 \mu \mathrm{m}$ band sources indicate relatively large clusters with attached sidegroups. The conditions around the cooler post-AGB objects seem conducive to the survival of these structures, which are probably rapidly destroyed in the harsh environment encountered on entering the PN phase. Magazzu \& Strazzulla (Poster III-92) have shown that the $3.4 \mu \mathrm{m}$ feature in the spectrum of the planetary nebula $\mathrm{Hb} 5$ is also unlikely to be a hot band, since although $\mathrm{F}(3.4) / \mathrm{F}(3.3)$ is quite high, there is no $\mathrm{v}=2-0$ feature at $1.67 \mu \mathrm{m}$ corresponding to the sum of the energies of the $3.3 \mu \mathrm{m}$ and $3.4 \mu \mathrm{m}$ features. The $3.4 \mu \mathrm{m}$ emission band therefore appears to be due to aliphatic C-H stretch vibrations. Lequeux \& Jourdain de Muizon (1990) found a broad $3.4 \mu \mathrm{m}$ absorption band (with $\tau=0.09$ ) in the spectrum of the C-rich postAGB object GL 618 and attributed it to aliphatic $\mathrm{C}-\mathrm{H}$ stretch vibrations of the kind invoked to explain similar absorption features arising in interstellar sightlines, such as that to the Galactic Centre.

\subsection{OXYGEN-RICH OBJECTS}

Hrivnak et al. (1989) have shown that the thermal infrared portion of the doublepeaked energy distributions of many oxygen-rich post-AGB objects can be adequately fitted by cool classical silicate emission. However, the energy distributions of a few O-rich post-AGB objects are not so easily fitted. Justtanont et al. (1992) have presented 10 and $20 \mu \mathrm{m}$ spectra of HD 161796 (F3Ib) and HD 179821=GL 2343 (G5Ia). There is a broad emission feature between 10 and $12 \mu \mathrm{m}$ in the spectra of both objects, which can be fitted very well by radiation-disordered olivine. The mid-IR energy distribution of both objects shows a $13-16 \mu \mathrm{m}$ rise that is too steep to be fitted by any combination of cool continuum plus silicate and Justtanont et al. (1992) argued that the rise could be explained by iron oxides of the type invoked by Cox (1990) to explain a similar steep rise seen in the IRAS LRS spectra of a number of compact $\mathrm{H}$ II regions.

Forveille et al. (1987) noticed that the source IRAS $09371+1212$ has unique IRAS colours, showing a huge peak in the $60 \mu \mathrm{m}$ band. They found the source to be associated with $\mathrm{CO}$ emission, and with an optical reflection nebula showing an M4 spectrum. They suggested that it was a post-AGB object with the peak in the $60 \mu \mathrm{m}$ band being due to emission in the $40-70 \mu \mathrm{m}$ bands of water ice and christened it the 'Frosty Leo Nebula'. Their suggestion of the presence of ice was soon confirmed by Rouan et al. (1988), who found a deep ice absorption band at $3.1 \mu \mathrm{m}$ in its spectrum. Omont et al. (1990) obtained a $35-70 \mu \mathrm{m} \mathrm{KAO}$ spectrum of IRAS $09371+1212$, which confirmed that it had prominent ice emission features at $44 \mu \mathrm{m}$ and $62 \mu \mathrm{m}$. The presence of the $62 \mu \mathrm{m}$ feature implied that the ice had to be crystalline rather than amorphous and their modelling yielded a best-fit grain temperature of $47 \mathrm{~K}$. The $44 \mu \mathrm{m}$ ice band was also detected in emission in the spectra of two other cool O-rich post-AGB objects, $\mathrm{OH} 127.8+0.0$ and $\mathrm{OH} 231.8+4.2$. 
There are a number of $\mathrm{PN}$ with spectral characteristics which indicate the simultaneous presence of C-rich and O-rich material. IRAS 07027-7934 was identified by Menzies \& Wolstencroft (1990) with a young planetary nebula having a cool carbonrich WC central star and they noted that its LRS spectrum showed the UIR bands. While this is indicative of a C-rich nebula, Zijlstra et al. (1991) found that it also had a strong $\mathrm{OH}$ maser, normally indicative of O-rich material. The Type I bipolar planetary nebula NGC 6302 has a spectrum which shows the $8.7 \mu \mathrm{m}$ and $11.3 \mu \mathrm{m}$ UIR bands (Roche \& Aitken 1986) but additionally has a weak OH maser (Payne, Phillips \& Terzian 1988). NGC 6302 also has a prominent $18-19 \mu \mathrm{m}$ silicate emission feature (Barlow, Skinner \& Justtanont, unpublished).

A possible interpretation of the presence of materials indicative of both O-rich and C-rich environments is as follows. The $\mathrm{C}+\mathrm{N}$ abundance derived for NGC 6302 by Aller et al. (1981) is the same as the mean for the non-Type I ('standard') planetary nebulae in their Table 4 and its $\mathrm{O}$ abundance is also the same as the mean value found for standard PN. In standard PN, carbon is greatly enhanced by the third dredge-up, which brings up primary carbon from the helium-burning shell, while in NGC 6302 it is nitrogen which is greatly enhanced. Since the first and second dredge-ups merely recycle CNO via the CN and CNO cycles (Iben \& Renzini 1983), it is clear that the third dredge-up must have occurred in NGC 6302 too, followed by envelope-burning (a process whereby dredged-up carbon is converted to nitrogen by the $\mathrm{CN}$ cycle at the bottom of the hydrogen envelope of sufficiently massive AGB stars; Iben \& Renzini 1983). Carbon dredge-ups, which can take the $\mathrm{C} / \mathrm{O}$ ratio to above unity, are nearly instantaneous but it takes more time for envelope burning to convert sufficient $\mathrm{C}$ to $\mathrm{N}$ to drop the surface $\mathrm{C} / \mathrm{O}$ ratio to below unity. Therefore between successive carbon dredge-ups there can be a Crich envelope phase followed by an O-rich envelope phase, with carbon-rich grains and oxygen-rich grains being alternately synthesised in the AGB star's outflow. Both types of grains can then be observed later in the object's evolution.

\section{References}

Allamandola, L.J., Tielens, A.G.G.M. and Barker, J.R.: 1989, ApJS, 71, 733

Aller, L.H., Ross, J.E., O'Mara, B.J. and Keyes, C.D.: 1981, MNRAS, 197, 95

Ashley, M.C.B., 1990, Proc. Astr. Soc. Austral., 8, 360

Barlow, M.J.: 1983, in Proc. IAU Symp. No. 103, Planetary Nebulae, ed. D.R. Flower, Reidel, 105

Borkowski, K.J. and Harrington, J.P.: 1991, ApJ, 379, 168

Buss, R.H. et al.: 1990, ApJ, L23

Cohen M., et al.: 1989, ApJ, 341, 246

Cox, P.: 1990, $A \& A, \mathbf{2 3 6}$, L29

de Muizon, M., Geballe, T.R., d'Hendecourt, L.B. and Baas, F.: 1986, ApJ, 306, L105

Duley, W.W.: 1985, MNRAS, 215, 259

Forveille, T., Morris, M., Omont, A. and Likkel, L.: 1987, $A \& A$, 176, L13

Forrest, W.J., Houck, J.R. and McCarthy, J.F.: 1981, ApJ, 248, 195

Furton, D.G. and Witt, A.N.: 1990, ApJ, 364, L45

Furton, D.G. and Witt, A.N.: 1992, ApJ, 386, 587

Geballe, T.R., Tielens, A.G.G.M., Allamandola, L.J., Moorhouse, A. and Brand, P.W.J.L.: 1989, A p J, 341, 278

Geballe, T.R., Tielens, A.G.G.M., Kwok, S. and Hrivnak, B.J.: 1992, ApJ, 387, L89 
Geballe, T.R. and van der Veen, W.E.C.J.: 1990, $A \& A$, 235, L9

Gillett, F.C., et al.: 1989, $A p J$, 338, 862

Goebel, J.H. and Moseley, S.H.: 1985, $A p J, 290$, L35

Harrington, J.P., Monk, D.J. and Clegg, R.E.S.: 1988, MNRAS, 231, 577

Hoare, M.G.: 1990, MNRAS, 244, 193, 1990

Hoare, M.G., Roche, P.F. and Clegg, R.E.S.: 1992, MNRAS, 258, 257

Hora, J.L., Deutsch, L.K., Hoffman, W.F. and Fazio, G.G.: 1990, ApJ, 353, 549

Hrivnak, B.J., Kwok, S. and Volk, K.M.: 1989, ApJ, 346, 265

Iben, I. and Renzini, A.: 1983, Ann. Rev. Astr. Astrophys., 21, 271

Justtanont, K., Barlow, M.J., Skinner, C.J. and Tielens, A.G.G.M.: 1992, ApJ, 392, L75

Kwok, S., Volk, K.M. and Hrivnak, B.J.: 1989, $A p J$, 345, L51

Lequeux, J. and Jourdain de Muizon, M.: 1990, $A \& A$, 240, L19

Manchado, A., Pottasch, S.R., Garcia-Lario, P., Esteban, C. and Mampaso, A.: 1989, A EA A, 214, 139

Mathis, J.S., Rumpl, W. and Nordsieck, K.H.: 1977, $A p J$, 217, 425 (MRN)

Menzies, J.W. and Wolstencroft, R.D.: 1990, MNRAS, 247, 177

Middlemass, D.: 1988, MNRAS, 231, 1025

Middlemass, D.: 1990, MNRAS, 244, 294

Middlemass, D., Clegg, R.E.S. and Walsh, J.R.: 1989, MNRAS, 239, 5P

Moseley, S.H. and Silverberg, R.F.: 1986, in Interrelationships among Circumstellar, Interstellar and Interplanetary Dust, ed. J.A. Nuth and R.E. Stencel, NASA CP-2403, A18

Nagata, T., et al.: 1988, $A p J$, 326, 157

Omont, A.: 1993, in Astronomical Infrared Spectroscopy: Future Observational Directions, ed. S. Kwok, ASP Conf. Ser., in press

Omont, A., et al.: 1990, $A p J$, 355, L27

Payne, H.E., Phillips, J.A. and Terzian, Y.: 1988, ApJ, 326, 368

Roche, P.F. 1989, in Proc. IAU Symp. No. 131, Planetary Nebulae, ed. S. Torres-Peimbert, Kluwer Academic Publishers, 117

Roche, P.F. and Aitken, D.K.: 1986, MNRAS, 221, 63

Roche, P.F. Aitken, D.K. and Smith C.H.: 1991, MNRAS, 252, 282

Rouan, D., Omont, A., Lacombe, F. and Forveille, T.: 1988, $A \& A$, 189, L6

Schmidt, G.D., Cohen, M. and Margon, B.: 1980, $A p J$, 239, L133

Sourisseau, C., Coddens, G. and Papoular, R.: 1992, $A \& A$, 254, L1

Tokunaga, A.T., et al.: 1988, ApJ, 328, 709

van der Veen, W.E.C.J., Habing, H.J. and Geballe, T.R.: 1989, $A \& A, \mathbf{2 2 6}, 108$

Watson, W.D.: 1972, ApJ, 176, 103

Witteborn, F.C. et al.: 1989, $A p J, \mathbf{3 4 1}, 270$

Zijlstra, A.A., Gaylard, M.J., te Lintel Hekkert, P., Menzies, J., Nyman, L.-A. and Schwarz, H.E.: 1991, $A \& A, \mathbf{2 4 3}, \mathrm{L} 9$ 